\title{
Effect of Ascorbic Acid on Fruit Quality during Postharvest
}

\author{
Yuan $X Z^{1, a}$, Liang $D^{1,2, b}$, Wang $X^{1, c}$, Xia $H^{1,2, d^{*}}$ \\ ${ }^{1}$ College of Horticulture, Sichuan Agricultural University, Chengdu 611130, China; \\ 2 Institute of Pomology and Olericulture, Sichuan Agricultural University, Chengdu 611130, China \\ a1723065255@qq.com, b756141959@qq.com, c347434587@qq.com, d381683619@qq.com
}

\#Co-first author, *Corresponding author

\begin{abstract}
Key words: kiwifruit; ascorbic acid; postharvest storage
Abstract. The fruit of kiwi is rich in nutrients and is deeply loved by people. However, the storage period is not long, and the shelf period is short and easy to be soft. In this experiment, the effects of ascorbic acid on the post-harvest storage of kiwifruit were studied by measuring the quality of the fruit with a solution of $100 \mathrm{mmol} \cdot \mathrm{L}^{-1}$ ascorbic acid based on the fruit of 'Jinshi No. 1'. The results showed that ascorbic acid treatment reduced fruit quality, reduced fruit hardness and reduced weightlessness in 7-14 days compared with control (CK). Malonaldehyde showed a smaller decrease. Therefore, it is considered ASA treatment can improve fruit quality and antioxidant properties, and it is an alternative method for storage and preservation of kiwifruit after harvest.
\end{abstract}

\section{Introduction}

Kiwifruit is a kind of respiration-changing fruit, it has obvious physiological after-ripening process, and the post-harvest fruit is apt to soft rot. Therefore, the kiwifruit has the disadvantage of storage failure ${ }^{[1-2]}$. Kiwi fruit ripening and senescence are complex physiological and biochemical processes. This process includes the synthesis of ethylene, the degradation of cell walls, the softening of fruits, the hydrolysis of starch and the accumulation of sugar, the change of pulp color, and the formation of aromatic substances ${ }^{[3-5]}$.

Ascorbic acid is an important antioxidant substance in plants. It plays an important role in protecting cell membrane structure and reducing membrane lipid peroxidation. Many studies have shown that the appropriate concentration of AsA treatment can extend the shelf life of horticultural products such as crystal pears, apricots, grapes, litchi, and cherry tomatoes ${ }^{[6-9]}$.

In this test, $100 \mathrm{mmol} \cdot \mathrm{L}^{-1}$ ascorbic acid solution was used to treat kiwi fruit. And measured the weight loss, hardness, soluble sugar content, titratable acid content, malondialdehyde content and other physiological indicators of the treated fruits. Discuss the effect of this treatment on the storability of kiwi fruit and its relationship with storage and preservation technology, Provides theoretical basis for the research and development of kiwifruit storage and preservation technology.

\section{Materials and Methods}

Materials. 'Jinshi No. 1' kiwifruits used in this study were harvested from Kiwifruit Resource Orchard in Shifang $\left(104^{\circ} 16^{\prime} \mathrm{N}, 31^{\circ} 13^{\prime} \mathrm{E}\right)$, Chengdu, China. During the fruit harvest period, the fruit is harvested when the fruit's physiological maturity reaches (TSS $\geq 6-8$ ), and then transported back to the laboratory quickly after harvest. Select fresh fruit with uniform size, consistent maturity, no pests and diseases, and no mechanical damage as test materials.

Preparation of kiwifruit extracts. The selected kiwi fruit was immersed in a $5 \%$ hypochlorous acid solution for sterilization. After 10 minutes, they were randomly divided into two groups of 30 fruits. The first group was immersed in distilled water for 30 minutes as a control. The second group was soaked in $100 \mathrm{mmol} \cdot \mathrm{L}-1$ ascorbic acid for $30 \mathrm{~min}$. Put the soaked fruits in a cool and ventilated place to dry, then put them in a refrigerator at $4{ }^{\circ} \mathrm{C}$ for storage. Samples were taken 5 times, once every 7 days, and 6 kiwifruits were randomly selected each time. The removed fruits were diced and stored at $-80{ }^{\circ} \mathrm{C}$ for other indicators. 
Determination of indicators. Use electronic scales to measure single fruit weight; The hardness of the fruit was measured with a GY-3 type hardness meter. The soluble solids (TSS) was measured using ATAGO's PAL type sugar test; The titratable acid was determined by acid-base titration ${ }^{[10]}$. Malondialdehyde (MDA) content was determined using the thiobarbituric acid method ${ }^{[11]}$. The above indicators were repeatedly measured three times or more, and the average value was taken as the final data of each treatment.

Data analysis. Excel 2007 was used for data processing and graphing. Analysis of variance was performed by the SPSS software (SPSS, Inc., Chicago, IL, USA).

\section{Results and Analysis}

Weight loss rate of fruit. The decrease in post-harvest weight of fruit indicates the loss of nutrients such as starch. As can be seen from Figure 1, there was no significant difference in the weight loss rate in the first 7 days of storage between the experimental group (AsA group) and the control group (CK group); From $7 \mathrm{~d}$ to $21 \mathrm{~d}$, the weightlessness rate of CK increased rapidly. However, although the rate of weightlessness in the experimental group also increased, the rate of increase was relatively slow. However, on the 28th day, the rate of weight loss in the two groups reached agreement again.

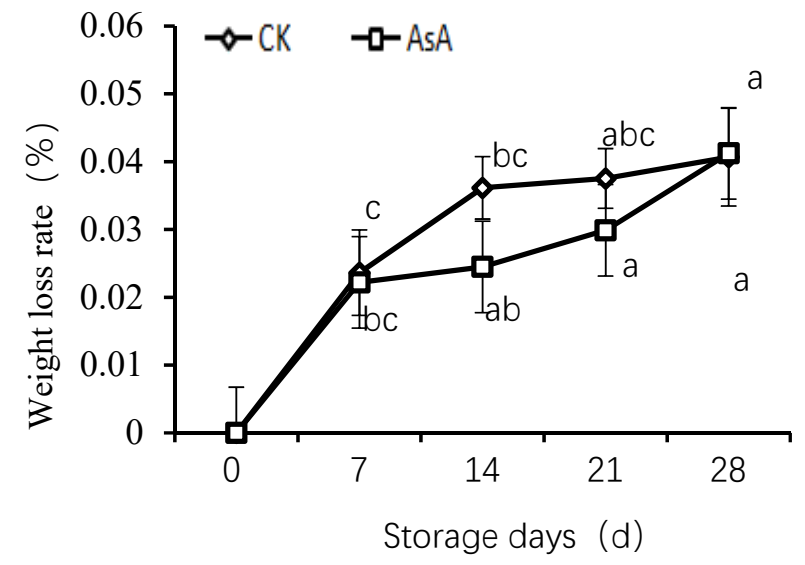

Fig 1 weight loss of kiwifruit during storage

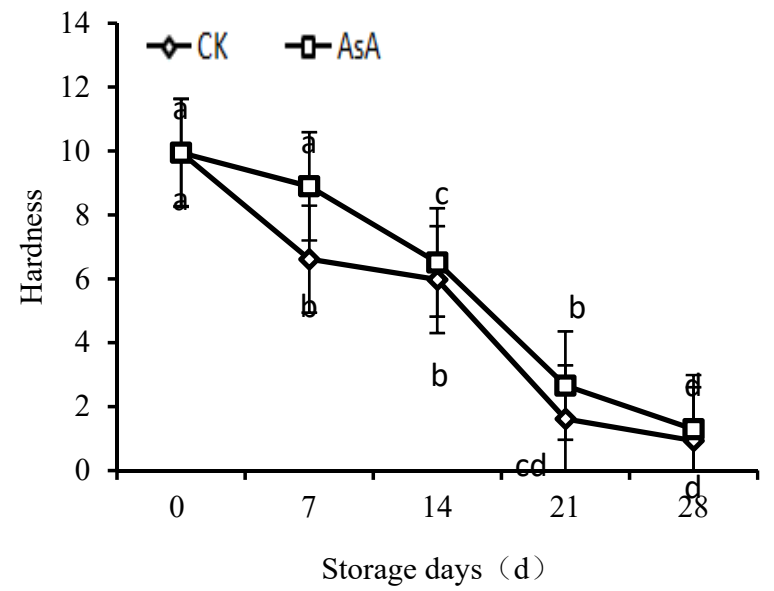

Fig 2 changes of hardness of kiwifruit during storage

Kiwifruit hardness. After the kiwi fruit was harvested, the pectin and starch materials in the fruit were gradually degraded, resulting in softening of the fruit and reduced hardness. As can be seen from Figure 2, in the first 7 days of storage, the hardness of the CK group decreased faster, while the hardness of the ASA group decreased more slowly. From the 7th to the 14th day, the decrease in the hardness of the control group slowed down, while that of the ASA group increased rapidly. On the 14th day, their hardness is approximately equal, After the 14th day, the two groups had the same rate of decline, and the 28th day hardness was approximately equal.

Soluble sugar. As can be seen from Figure 3, in the first 7 days after storage, soluble sugar rises in the CK group at a faster rate and has a higher content. On the 7th to the 14th day, the soluble sugar content in the CK group increased slowly and was slightly lower than that in the ASA group. From 14 days to 28 days, the two groups have the opposite trend. From the 14th to the 21 st day, the ASA group gradually decreased while the CK group gradually increased. From the 21st to the 28th day, the soluble sugar content of the CK group decreased, while the ASA group increased. Finally, on the 28th day, the soluble sugar content in both groups was approximately equal. 

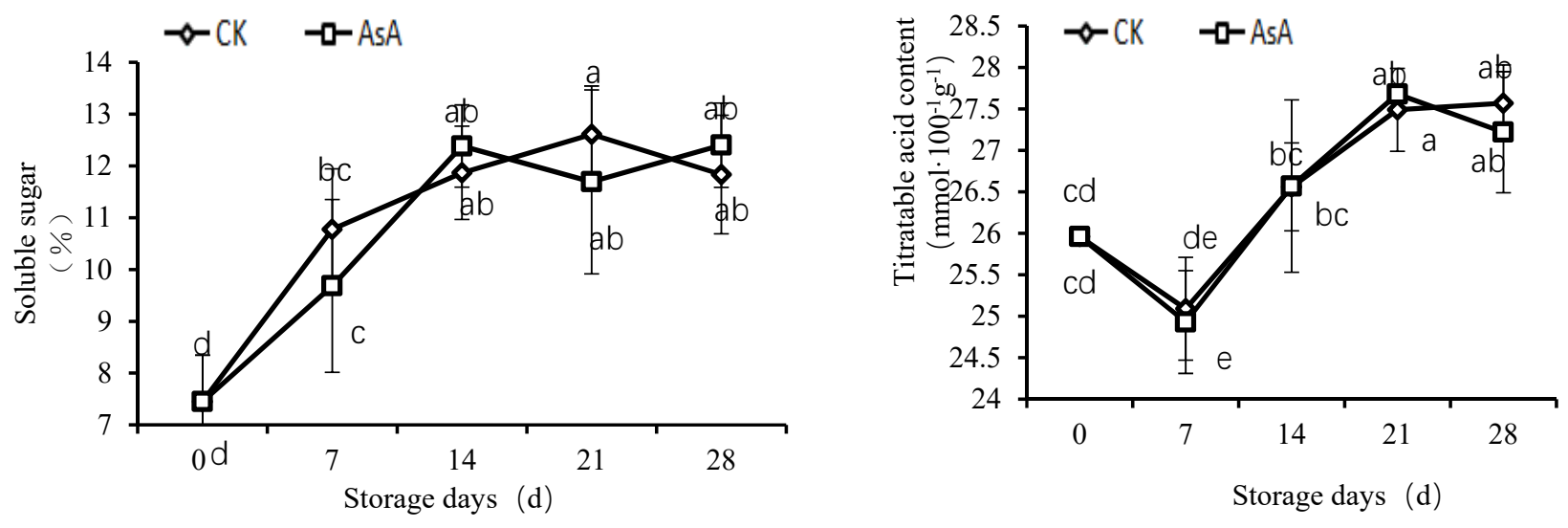

Fig. 3 sugar content of kiwifruit during storage

Fig 4 titrated acid content of kiwifruit during storage

Changes of titratable acid. As can be seen from Figure 4, the overall trend of changes in titratable acid content is the same in both groups. In the first 7 days, the titratable acid content decreased and the rate of decline was approximately equal. From $7 \mathrm{~d}$ to $21 \mathrm{~d}$, the titratable acid content increased significantly and the rate of change was approximately the same. From the 21 st to the 28th day, the ASA and CK groups fluctuate slightly. However, on the 28th day, the titratable acid content was similar in both groups.

Malondialdehyde in fruit. As shown in Figure 5, in the first 7 days, the MDA content in the AsA group decreased rapidly, while that in the CK group decreased slowly. From the 7th to the 14th day, the CK group decreased faster, while the ASA group decreased more slowly. However, the malondialdehyde content of the ASA group was still lower than that of the CK group. From the 14th to the 28th day, the CK group and the ASA group showed similar changes in the descending speed. On the 28th day after storage, the MDA content of the ASA group was still slightly lower than that of the CK group, but the difference was not significant.

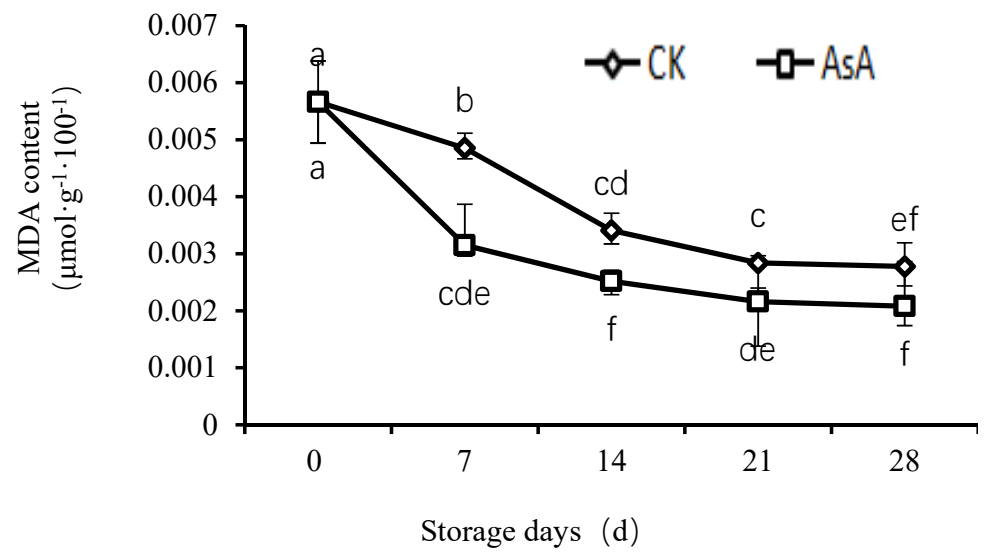

Fig 5 Effect of ascorbic acid on MDA content during storage of kiwifruit

\section{Conclusions}

The research on fruit softening, sugar acid amount, antioxidant capacity, etc. of kiwi fruit after ginseng production in Jinshi No. 1 can be used to improve the fruit ripening period or consumption period. MDA can reflect the degree of membrane lipid peroxidation, which indirectly shows the degree of damage to the membrane system and the plant's resistance to stress. The MDA content in the AsA group showed a rapid downward trend and was lower than CK. This shows that AsA treatment can reduce the degree of membrane peroxidation and protect cell membrane structure and function from damage.

The test showed that the treatment of $100 \mathrm{mmol} \cdot \mathrm{L}^{-1}$ ascorbic acid solution reduced the fruit quality, hardness, and weight loss rate. Therefore, it can be considered that treatment with ascorbic acid can improve fruit quality and anti-oxidation, and this treatment method is an effective method for post-harvest storage of kiwifruit. 


\section{Acknowledgements}

This work was financially supported by the Sichuan Technical Supports Foundation (2016NZ0105) and Training Programs of Innovation for Undergraduates in Sichuan (201610626057).

\section{References}

[1] Zhao S, Wang M, Yang G, et al. Assessment of Fruit Quality of Twelve Kiwifruit Varieties Introduced and Planted in China[J]. Research of Agricultural Modernization, 2002, 23(6):455-457.

[2] Wang S, Yang J, Duan C, et al. Advance of Research on Mature Physiological and Preservation Technology of Kiwifruit[J]. Chinese Agricultural Science Bulletin, 2013.

[3] Wang S, Yang J, Duan C, et al. Advance of Research on Mature Physiological and Preservation Technology of Kiwifruit[J]. Chinese Agricultural Science Bulletin, 2013.

[4] Chen J, Zeng R, Li P. Advance of Research on Postharvest Physiology of Kiwifruit and Its Storage Technology[J]. Acta Agriculturae Universitis Jiangxiensis, 2002, 24(4):477-483.

[5] Zhang H, Ma C, Peng X, et al. Advances of Research on Postharvest Physiology and Disease of Hongyang Kiwifruit[J]. Guangdong Chemical Industry, 2017.

[6] Cho J S, Jeong M C, Moon K D. Effects of Ultrasound and Ascorbic acid Cotreatment on Browning of Fresh-cut 'Tsugaru' Apples[J]. Maternal \& Child Nutrition, 2012, 19(3):323-327.

[7] Chun-Hua M A, Zhang M, Ming-Jun L I, et al. Influence of Exogenous Ascorbic Acid on Postharvest Ripening and AsA-GSH Cycles in Apple Fruit[J]. Journal of Yunnan Agricultural University, 2012.

[8] Liu K, Jing G, Yuan C, et al. Effect of Ascorbic Acid Treatment on Physiology and Antioxidants of Cherry Tomato Fruits during Storage[J]. Chinese Journal of Tropical Crops, 2012.

[9] Gao L, Xia T, Zhang H. Studies on the index of membrane lipid peroxidation--MDA during senescence of Kiwifruit[J]. Journal of Anhui Agricultural University, 2000. 